\title{
A Query Language Combining Object Features and Semantic Events for Surveillance Video Retrieval
}

\author{
Thi-Lan Le ${ }^{1,2}$, Monique Thonnat ${ }^{1}$, Alain Boucher ${ }^{3}$, and François Brémond ${ }^{1}$ \\ 1 ORION, INRIA, 2004 route des Lucioles, B.P. 93, 06902 Sophia Antipolis, France \\ \{Lan.Le_Thi, Monique.Thonnat, Francois.Bremond\}@sophia.inria.fr \\ ${ }^{2}$ International Research Center MICA Hanoi University of Technology, Viet Nam \\ 3 Equipe MSI, Institut de la Francophonie pour l'Informatique, Hanoi, Viet Nam \\ Alain.Boucher@auf .org
}

\begin{abstract}
In this paper, we propose a novel query language for video indexing and retrieval that (1) enables to make queries both at the image level and at the semantic level (2) enables the users to define their own scenarios based on semantic events and (3) retrieves videos with both exact matching and similarity matching. For a query language, four main issues must be addressed: data modeling, query formulation, query parsing and query matching. In this paper we focus and give contributions on data modeling, query formulation and query matching. We are currently using color histograms and SIFT features at the image level and 10 types of events at the semantic level. We have tested the proposed query language for the retrieval of surveillance videos of a metro station. In our experiments the database contains more than 200 indexed physical objects and 48 semantic events. The results using different types of queries are promising.
\end{abstract}

\section{Introduction}

Video surveillance is producing huge video databases. While there are many works dedicated to object detection, object tracking and event recognition [7], few works have been done for accessing these databases. For surveillance video indexing and retrieval, apart from object tracking, object classification and event recognition, we have 4 main issues: data modeling, query formulation, query parsing and query matching. The data modeling determines which features are extracted and how they are organized and stored in the database. The query formulation specifies the way in which the user expresses his/her query while the query parsing [5] specifies the way in which the system analyzes (parses) this query into an internal representation. The aim of query matching is to compare elements stored in the database with the query.

The first works dedicated for the surveillance video indexing and video concentrate on data modeling. In [7], IBM smart surveillance engine successfully detects moving objects, tracks multiple objects, classifies objects and events. 
However, for retrieving in the database, the queries are done based on only recognized events and metadata. In [6], a data model is built for online video surveillance. Various query types are also presented. In [3], Saykol et al. presented a framework and a visual surveillance querying language (VSQL) for surveillance video retrieval. These previous works have three main drawbacks. Firstly, they work with an assumption that in the indexing phase objects are perfectly tracked and events are perfectly recognized. It is the reason why video retrieving is based on the exact matching of semantic events and metadata. However, object detection and event recognition are not always successful. The video retrieving must work well under imperfect indexing. In this case, the similarity matching on object features is necessary. Secondly, these approaches limit the search space. Because the videos are only indexed by a set of recognized events, the users' queries are restricted to a limited set of predefined events. Thirdly, it is not flexible and does not take into account various users' interests and users' degrees of knowledge. The users could need more or less information according to their interest and could define differently an 'event' in the form of a query in function of their knowledge in this domain.

Our main contributions are designing a video data model and proposing a novel query language. Our data model is different from the model proposed in [6] because it contains object visual features. Our query language overcomes the previous works [7], [6], [3] because it (1) enables users to make queries both at the image level and at the semantic level (2) allows the users to define their own scenarios based on semantic events and (3) retrieves videos with both exact matching and similarity matching.

The rest of this paper is organized as follows: Section 2 describes the proposed approach including data model, query language and query matching. In section 3 , we describe some experimental results and their performance evaluation. We conclude this paper in section 4 .

\section{Proposed Approach}

Figure 1 shows the general architecture of the proposed approach. This approach is based on an external Video Analysis module and on two internal phases: an indexing phase and a retrieval phase. The external Video Analysis module performs tasks such as mobile object detection, mobile object tracking and event recognition. The results of this module are some Recognized Video Content. These Recognized Video Content can be physical objects, trajectories, events, scenarios, etc. So far, we are only using physical objects and events but the approach can be extended to other types of Recognized Video Content. The indexing phase takes results from the Video Analysis module as input data. The indexing phase has two main tasks: feature extraction and data indexing. It performs feature extraction to complete the input data by computing missing features and data indexing using a data model. The retrieval phase is divided into five main tasks: query formulation, query parsing, query matching, result ranking and result browsing. In the query formulation task, in order 


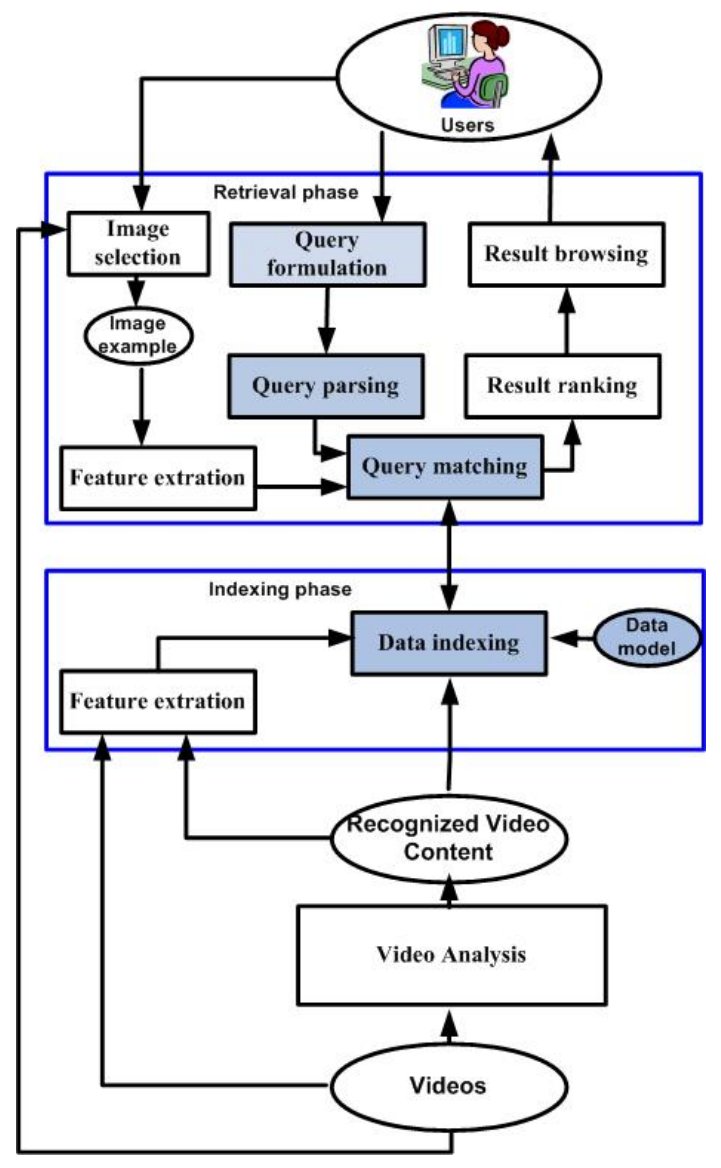

Fig. 1. Global architecture of our approach. This approach is based on an external Video Analysis module and on two internal phases: an indexing phase and a retrieval phase. The indexing phase takes results from the Video Analysis module as input data and performs feature extraction and data indexing using a data model. The retrieval phase takes queries from users (by the query formulation task), analyzes and evaluates them (by query parsing and query matching tasks) using indexed data in the indexing phase. The retrieval results are ranked and returned to the users (by the result ranking and the result browsing tasks). The focus of this paper concerns the parts in blue. 
to make the users feel familiar with the query language, we propose a SVSQL (Surveillance Video Structured Query Language) language. The vocabulary and the syntax are described in the next section. In the query, the users can select an image example global or a region in an image from the database (by the image selection task). In this case, the feature extraction task computes some features in the image example which are used by the query matching task. In the query parsing task, queries built with the proposed language are transmitted to a parser. This parser checks the vocabulary, analyzes the syntax and separates the query into several parts. The query matching task searches in the database the elements that satisfy the query. The obtained results are ranked and returned to the users.

\subsection{Data model}

Our data model contains two main components of interest form the user's point of view: physical objects and events.

Physical objects: they are the detected objects in the video database. A physical object can be a static object (e.g. contextual object) or a moving object (e.g. a person, a vehicle). Let $\mathrm{P}$ be a physical object, $\mathrm{P}$ is defined as follows: $P$ $=(I d$, [Type], [Name], 2D_positions, 3D_positions, MBRs (Minimum Bounding Box), Features, Time_interval) where $I d$ is the label of the object, the Type and Name attributes are optional. The 2D_positions, 3D_positions, MBRs are the sets of 2D positions, 3D positions, MBRs of this object during its lifetime indicated by Time_interval. The Features currently available in the system are the color histogram and a set of detected keypoints by using SIFT (Scale Invariant Feature Transform) descriptors. These features are computed by the Feature extraction task. We use the SIFT descriptors because the SIFT descriptors are invariant to image scale and rotation and they are shown to provide robust matching across a substantial range of affine distortion change in $3 \mathrm{D}$ view point, addition of noise, and change in illumination. Therefore, they help to match efficiently images. However, SIFT descriptors do not focus on color information like the color histogram. The methods for extracting these features can be found in [4] for the color histogram and in [1] for the SIFT descriptors.

Events: They are the recognized events in the video database. Let $\mathrm{E}$ be an event, $\mathrm{E}$ is defined as follows: $E=(I d$, Name, Confidence_value, Involved_Physical_objects, [Sub_events], Time_interval). Where Id is the label of the event and Name is the name of the event. The Confidence_value specifies the confidence degree of recognized event. The work presented in Section 2 does not take into account this information. In our work, the Confidence_value is used to compute the final distance between video frames and the query. The Involved_Physical_objects specifies which physical objects are involved in this event, while the Sub_events are optional, and the Time_interval indicates the frames in which the event is recognized.

The advantage of our data model is that it is independent of any application and of any feature extraction, learning and event recognition algorithms. Therefore, we can combine results of different algorithms for feature extraction, 
learning and event recognition (both descriptive and stochastic approaches) and use it for different application domains.

\subsection{Proposed language and query syntax}

The syntax of a query expressed by our language is the following:

$$
\text { SELECT }<\text { Output }>\text { FROM }<\text { Database }>\text { WHERE }<\text { Condition }>
$$

Where: SELECT, FROM, WHERE are keywords for a query and they are mandatory. A graphic interface can be developed to generate this syntax but it is out of the scope of this paper.

- Output specifies the format of the retrieved results. It can have two values: one is video_frames indicating that the retrieved results are video frames in which the <Condition $>$ is satisfied and the other is number_of_events indicating that the retrieved result is the number of events in the database satisfying the $<$ Condition $>$.

- Database specifies which parts of the video database are used to check the $<$ Condition $>$. It can be either $*$ for the whole video database or a list of named subparts. This is interesting for surveillance because the video database can be divided into several parts according to time or location. It allows to accelerate the retrieval phase in the case that the users know already which parts of the video database they are interested in.

- Condition specifies the conditions that the retrieved results must satisfy. The users express their requirements by defining this component. The condition may have more than one expression connected together by logic operators (AND, OR, NOT), each expression is started by "(" and ended by ")". This component is the most important component in the language.

There are two types of expression in the condition component: a declaration expression $\left(\alpha_{d}\right)$ which is mandatory and a constraint expression $\left(\alpha_{r}\right)$ which provides additional conditions. The declaration expression indicates the types of variable while the constraint expression specifies constraints the variable must satisfy.

The syntax for a declaration expression is: $(v:$ type $)$ where $v$ is a variable. It is the place where the user specifies if the retrieval is at the image level, the semantic level or both levels. The authorized types are : Physical_object and its subtypes (Person, Group, Luggage) and Event. In image and video retrieval applications, users usually want to retrieve indexed data that is similar to an example they have. Therefore, besides Physical objects and Events, we add another type SubImage. SubImage has Features attribute like the Physical objects. In query, ( $\mathrm{v}$ : SubImage) means that $\mathrm{v}$ will be set by users image example.

The syntax for a constraint expression is very rich. The constraint expression can be expressed by using a set of projections, functions, predicates, algebra operators and constants. Currently, the authorized projections are \{'s $I d$, 's Type, 's Name, 's 2D_positions, 's 3D_positions, 's MBRs, 's Features, 's 
Time_interval $\}$ for physical object and $\{$ 's Id, 's Name, 's Confidence_value, 's Involved_Physical_objects, 's Sub_events, 's Time_interval $\}$ for event; there are two authorized functions which are histogram_distance that returns the distance between color histograms and number_matched_keypoint that returns the number of matched keypoints between an example image and an indexed object; there are four authorized predicates which are color_similarity and keypoints_matching that return true if an image example and an indexed object are similar in term of color histogram or keypoints, involved_in which verifies whether one indexed object belongs to an event and before which verifies whether an event occurs before another event; the authorized algebra operators are $=,<,>,>=,=<$, $!=\}$; the constants can be either numbers or strings.

This language is rich enough to express numerous possible queries. Based on the technique proposed in [5], we implement a parser to check automatically the syntax of each query. This parser automatically analyzes the syntax of the query. The results of this parsing allow to locate which databases will be used to match query, which variables must be set and which results must be returned.

An example expressed by this language at the semantic level is: Find Close_to_Gates events occurring in videos of all databases.

SELECT video_frames FROM * WHERE ((e: Events) AND (e's Name = "Close_to_Gates"))

where $e$ is a variable of Events, $e^{\prime} s$ Name gets Name of $e$.

Another example expressed by this language at the image level is: Find indexed persons in the database named Video_Database that are similar to a given image.

SELECT video_frames FROM Video_Database WHERE ((p: Person) AND ( $i$ : SubImage) AND (i color_similarity $p)$ )

where $p$ is a variable of Person, $i$ is a variable that will be set by an image example, color_similarity is predicate that decide whether two images are similar (in color).

\subsection{Query matching}

For each expression $\alpha$ in the condition field, the evaluation of the expression $\alpha$ is performed by matching the indexed database $\mathrm{D}$ and the expression $\alpha$. The results of this process are a set of physical objects and events extracted from $\mathrm{D}$ that satisfy $\alpha$. Let $\eta_{i}=\left\{\varsigma_{i}, \mu_{i}, I_{i}\right\}$ be the ith result instance of the expression $\alpha$ and $\eta$ be the set of the result instances of $\alpha$ where $\varsigma_{i}$ are the physical objects or the events, $\mu_{i}$ is the similarity degree that determines how much $\varsigma_{i}$ satisfy $\alpha$ in a time interval $I_{i}$. Currently, we have defined the similarity degree for the predicates based on the feature similarity (e.g. nearest neighbors for SIFT descriptors and histogram intersection for color histograms). With the other types the default value of the similarity degree is set to 1 .

If the query has more than one expression in the condition, the similarity degrees are computed according to the operator linking these expressions as follows: $\mu=\min \left(\mu_{j}, \mu_{k}\right), \mu=\max \left(\mu_{j}, \mu_{k}\right), \mu=\left(1-\mu_{k}\right)$ for AND, OR, NOT operators where $\mu_{j}, \mu_{k}$ are the similarity degrees of the expressions $\alpha_{j}, \alpha_{k}$ respectively. 


\section{Implementation and Experimental Results}

\subsection{Video event database}

In order to validate our approach, we have used two videos of 10 minutes and 2 hours acquired by two fixed cameras at different positions that record human activities in a metro station. An example of two scenes in two videos is shown in Fig.2. The scene contains a platform and several gates. The Video Analysis such as automatic object tracking, object classification and event recognition proposed in [5] have been automatically applied to these videos. As results, we have 221 indexed physical objects (101 for the first video and 120 for the second one) with their labels, 3D positions, 2D positions, MBRs and time intervals. The 120 physical objects in the second video are classified as 29 persons, 27 groups, 16 crowds, 25 luggages et 23 unknowns. One physical object is perfectly tracked and recognized if in all frames in which this object appears, this object is detected and has one sole label. In addition, 10 event types have been defined and recognized for each frame (inside Platform, close to Gates i (i from 1 to 9)) for the second video. These events are defined in the language proposed in [5] as follows:

Event $($ close_to,

PhysicalObjects((p : Person), (eq : Equipment))

Constraints ( $(p$ distance $e q \leq$ Close_Distance $))$

where eq is Gate $\mathrm{i}$ (i from 1 to 9 ), Close_Distance is a threshold.

Event(inside_zone,

PhysicalObjects((p : Person), (z:Zone))

Constraints ( $(p$ in $z))$

where $\mathrm{z}$ is a Platform, in is a predicate that checks whether p's center belongs to the polygon $\mathrm{z}$.

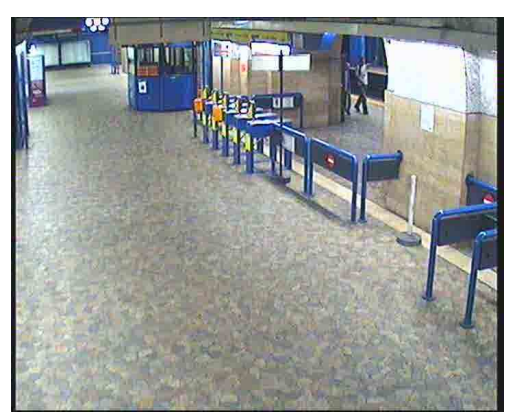

(a)

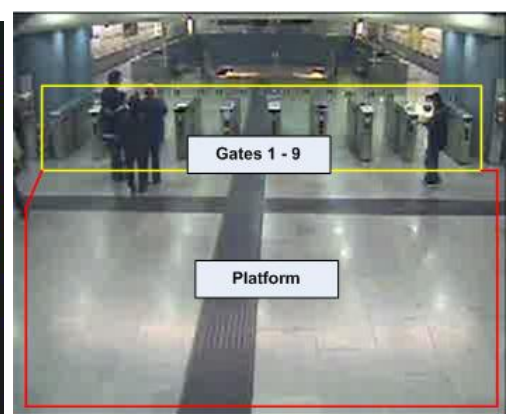

(b)

Fig. 2. Two scenes describe human activities in some metro stations(a) in the first video. (b) in the second video. 


\subsection{Experimental results and performance evaluation}

In order to evaluate the retrieval performance, we use the average normalized rank proposed in [2]. We do not use the measures of true positives, true negatives, false positives and false negatives like in classification problem because for the retrieval problem, the retrieval algorithm is good if it return relevant results first.

$$
\widetilde{\operatorname{Rank}}=\frac{1}{N N_{\text {rel }}}\left(\sum_{i=1}^{N_{r e l}}\left(R_{i}\right)-\frac{N_{r e l}\left(N_{\text {rel }}+1\right)}{2}\right)
$$

where $N_{r e l}$ is the number of relevant result for a particular query, $\mathrm{N}$ is the size of the tested set, and $R_{i}$ is the rank of the ith relevant result. $\widetilde{R a n k}$ is zero if all $N_{r e l}$ are returned first. The $\widetilde{R a n k}$ measure lies in the range 0 (good retrieval) to 1 (bad retrieval), with 0.5 corresponding to a random retrieval.

Experiment 1: The goal of this experiment is to check whether our proposed language enables users to retrieve effectively persons in the database even though they are not successfully detected and tracked. For this experiment, the query is: Find in the second video the video frames having persons that are similar (in term of keypoint matching) to the person in this example image. This query is expressed as follows:

SELECT video_frames FROM Video2 WHERE ((i: SubImage) AND (o: Person) $A N D$ (i keypoints_matching o))

where keypoints_matching is a predefined predicate of our language, $i$ is an example image.

Figure 3.a shows the average normalized rank for this experiment. There are 120 indexed persons in the second video. The retrieval performance is measured over all 120 images using each in turn as a query. Each image query has from 3 to 5 relevant images. The small obtained average normalized ranks (the maximum value being 0.3183 ) show that the proposed approach retrieve successfully the indexed objects even when they are imperfectly indexed.

Experiment 2: This experiment aims at pointing out the advantage of our language. It allows to retrieve interesting events with a detailed description. For instance, the event close_to_Gate1 (p) indicates that person $\mathrm{p}$ is close to the Gate 1. In the case of many close_to_Gate1 recognized instances, the user may be interested in only close_to_Gate1 frames containing a person that is similar to a given example. The user can ask a query as follows:

SELECT video_frames FROM Video2 WHERE ((i: SubImage) AND (o: Person) $A N D$ (e: Event) $A N D$ (e's Name $=$ close_to_Gate1) $A N D$ (o involved_in e) $A N D$ (i keypoints_matching o))

where $i$ is an image example, 's Name is a predefined projection of event's name, involved_in is a predefined predicate that determines whether one person is involved in an event and keypoints_matching is a predefined predicate described in section 2 .

To answer this query, the persons involved in all close_to_Gate1 events are used for keypoints_matching with the given example. The returned results for each query is a list of close_to_Gate1 events ranked by the number of matched 


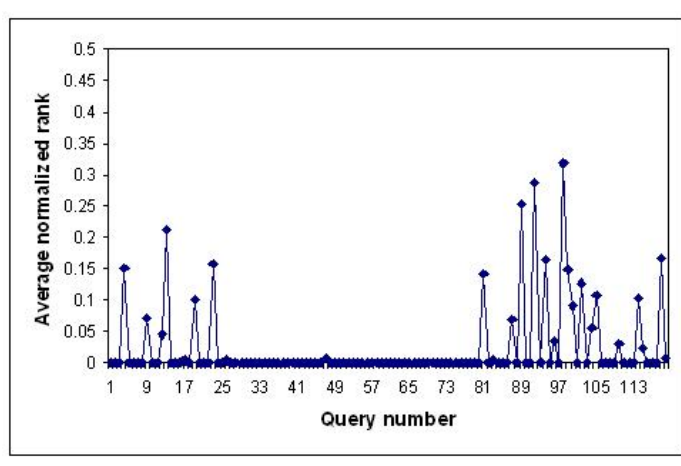

(a)

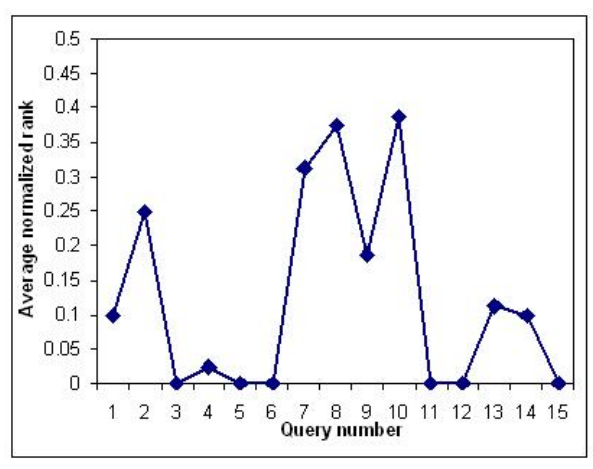

(b)

Fig. 3. (a) The average normalized rank for experiment 1 over 120 queries. (b) The average normalized rank for experiment 2 over 15 queries. The value 0 of average normalized ranks corresponds to good retrieval, value 1 corresponds to bad retrieval and 0.5 corresponds to random retrieval.

keypoints between the involved persons and the given example. Among 19 close_to_Gate1 events of the second video, there are several events concerning one sole person. We have 15 distinct persons concerned to these 19 events. For each person, we have chosen one image example. Totally, we have 15 example images. Each image turns as input for the query. Figure 3.b gives the obtained average normalized rank over 15 image examples and 19 events of close_to_Gate1 in the second video. The ground truth is made by hand for these 15 queries. A returned result is considered relevant if it is a close_to_Gate1 event whose the involved persons show the same person as in the given image example.

Experiment 3: The objective of this experiment is to measure the capacity of this language to define and retrieve new events from the recognized ones. From two recognized events in the database: inside_zone_Platform and close_to_Gate1, the user may write a query such as: Find the frames in which one person is going from the Platform to Gate1.

This query is expressed as follows:

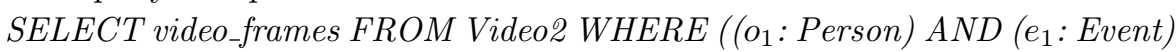
$A N D\left(e_{2}\right.$ : Event) AND (o involved_in $\left.e_{1}\right)$ AND (o $o_{1}$ involved_in $\left.e_{2}\right) A N D\left(e_{1}\right.$ 's Name $=$ inside_zone_Platform) $A N D$ (e $e_{2}$ s Name = close_to_Gate1) AND (e 1 before $\left.e_{2}\right)$ )

This query is automatically analyzed by the parser presented in the section 2.

Because of imperfect indexing, one person in the real world may be indexed as different persons within the database. Thanks to similarity matching returned results must consider all inside_zone_Platform and close_to_Gate1 events containing indexed persons that are similar (an exact matching that matches the indexed persons by their labels would have returned for this query incomplete results and sometimes empty ones as shown in Figure 4.a). With our technique 
to answer this query, the system first matches the involved persons in both inside_zone_Platform and close_to_Gate1 by keypoint matching. For each per-

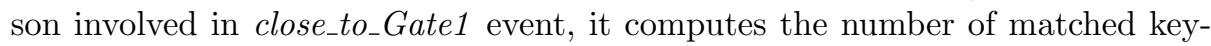
points between this person and the persons involved in the inside_zone_Platform event. A set of persons ordered by their matched keypoints are returned. The inside_zone_Platform events containing these persons become candidate for retrieval results. Then, these events are used to check whether they satisfy the before constraint with the close_to_Gate1 events. The before constraint performs based on the starting frames and the ending frames of inside_zone_Platform and close_to_Gate1 events.

For each $c l o s e_{-} t o_{-}$Gate 1 event, the retrieval result is a list of inside_zone_Platform events that satisfy the before constraint with close_to_Gate1 event and that are ranked by the number of matched keypoint between their involved persons and the person involved in close_to_Gate1 event. The returned result is considered relevant if it contains an inside_zone_Platform event that satisfies the before constraint and if their involved persons show the same person in the real world.

The average normalized rank for this experiment is given in Fig.4.b over 19 events of close_to_Gate1.

This experiment shows the capacity of this language to define new event from the recognized ones with satisfying results (average normalized ranks of all 19 events are smaller than 0.3).

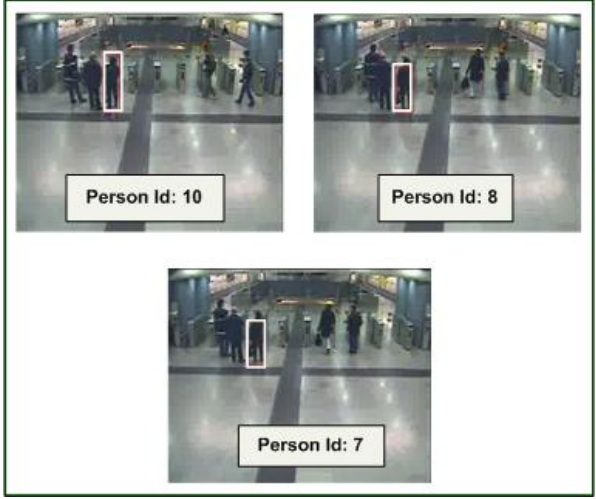

(a)

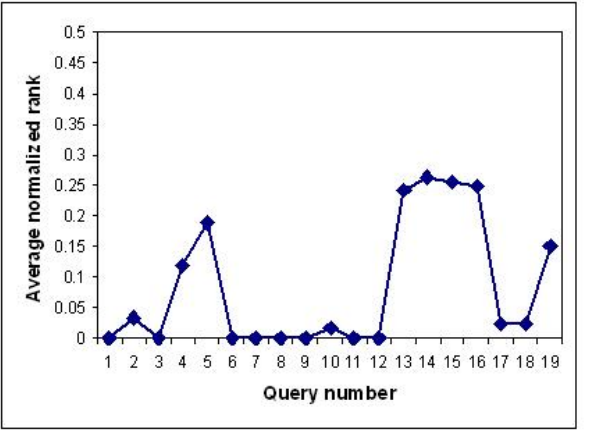

(b)

Fig. 4. (a)Three indexed persons with labels 10, 8, 7 describe the same person in the real world. These indexed persons belong to close_to_Gate1 and inside_zone_Platform events that satisfy before constraint. The exact matching based on persons' label gives only one result of person label 10 while our similarity matching gives three persons label 10, 8, 7 with respectively 1, 2, 5 of rank (b) The average normalized rank for experiment 3 over 19 close_to_Gate1 events. The value 0 of average normalized ranks corresponds to good retrieval, value 1 corresponds to bad retrieval and 0.5 corresponds to random retrieval. 
As shown in Fig.3 and Fig.4, most of the average normalized ranks of the three experiments are small but there are some cases where these measures are quite high (maximum value is respectively $0.3183,0.3875,0.262$ for the experiment 1, the experiment 2 and the experiment 3 ) because of the error in object tracking. Therefore, in addition of keypoints and color histogram we intend to use more features or to use a combination of features to solve these problems.

The experiments 1, 2 and 3 show that the proposed approach overcomes the first and the second drawback presented in the introduction. We present another example of query to explain how the proposed approach can do to overcome the third drawback. In the first video, we do not have the results of event recognition. Users may define a new Close to Gates event by stating query as follows:

SELECT video_frames FROM Video1 WHERE ((o: Person) AND (z: Gates) AND (o distance $z<$ threshold))

One person is close to gates if the distance between this person and the gates is smaller than a given threshold. The distance is computed based on the 3D position of the persons and the gates. This query takes into account users interest by using a threshold. User can set the value of a threshold as he/she wants. By setting two different values for the threshold, 100 and 150, we have two different results. The first result returns 10 indexed persons with 320 recognized instance of the Close to Gates event. The second one returns 20 indexed persons with 727 recognized instances.

\section{Conclusions}

In this paper, we have proposed an approach for video indexing and retrieval for surveillance based on a query language. This new language enables both image and semantic queries and similarity matching. The obtained results for three experiments show that: combining the image level and the semantic level (in experiment 2 and 3 ) and similarity matching (in experiment 1,2 and 3) manage imperfect object tracking and imperfect event recognition. New events defined by the user from the recognized ones have been successfully retrieved (in the experiment 3).

Currently, similarity matching has been limited to the color histogram and the keypoints. We plan to study and use more features to enrich the proposed language. In addition, the users may want to make a complex query containing several subqueries. How to combine the results from these subqueries is an issue that we plan to study in the future.

\section{References}

1. Lowe, D.G.: Distinctive image features from scale-invariant keypoints. International Journal of Computer Vision, Vol. 60 No. 2, 2004, 91-110.

2. Müller, H., Marchand-Maillet, S., Pun, T.: The truth about corel - evaluation in image retrieval. In Proc. Of. CIVR, London, July 2002, 28-49. 
3. Saykol, E., Güdükbay, U., Ulusoy, O.: A database model for querying visual surveillance by integrating semantic and low-level features. In Proc. of 11th International Workshop on Multimedia Information Systems (MIS05)), Vol. 3665, Sorrento, Italy, September 19-21 2005, 163-176.

4. Swain, M. J., Ballard, D.H.: Color indexing. International Journal of Computer Vision, Vol. 7, No. 1, 1991, 11-32.

5. Vu, V.T., Brémond, F., Thonnat, M.: Automatic video interpretation: A novel algorithm for temporal scenario recognition. In Proc. of International Joint Conference on Artificial Intelligence (IJCAI03), Acapulco, Mexico, August 9-15 2003, 1295-1302.

6. Durak, N., Yazici, A., George, R.: Online Surveillance Video Archive System. In Proc. of International Multimedia Modeling Conference, Singapore, January 2007, 376-385.

7. Hampapur, A., Brown, L., Connell, J., Ekin, A., Haas, N., Lu, M. Merki, H., Pankanti, S., Senior, A., Shu, C., Tian, Y. L.: Smart Video Surveillance: Exploring the concept of multiscale spatiotemporal tracking. In IEEE Signal Processing Magazine, Vol. 22, Issue 2, March 2005, 38-51. 